\title{
Minimal classical communication and measurement complexity for quantum information splitting of a two qubit state
}

\author{
Siddharth Karumanchi \\ Birla Institute of Technology and Science, Pilani, Rajasthan- 333031, India \\ Sreraman Muralidharan* \\ Loyola College, Nungambakkam, Chennai - 600 034, India \\ Prasanta K. Panigrahi甲 \\ Indian Institute of Science Education and Research (IISER) Kolkata, Salt Lake, Kolkata - 700106, India and \\ Physical Research Laboratory, Navrangpura, Ahmedabad - 380 009, India
}

\begin{abstract}
We provide explicit schemes for quantum information splitting (QIS) of real or equatorial two qubit states among two parties, through the five particle cluster and Brown states respectively. The schemes introduced are neither fully deterministic nor completely probabilistic and are carried out in the case where, the information that is to be shared is partially or fully known to the sender. We show that, QIS can be accomplished with just two classical bits, as against four classical bits, when the information is unknown to the sender.
\end{abstract}

PACS numbers: 03.67.Hk, 03.65.Ud

Keywords: Entanglement, Teleportation, State sharing, Superdense coding

\section{INTRODUCTION}

Entanglement is the most striking and counter intuitive feature of quantum mechanics that has come in handy for a number of practical applications in communication technology [1]. It is well understood only in the case of two particles. In the multiparticle scenario, much remains to be understood and explored owing to the increase in complexity with the number of qubits as there are numerous ways in which they can be entangled. Entangled states are used as a communication resource in teleportation, secret sharing and superdense coding. Quantum teleportation is a technique for transfer of information between parties, using a distributed entangled state and a classical communication channel. In a remarkable work, Bennett et al. [2] showed that, an EPR pair, could be used for the teleportation of an unknown single qubit state $\alpha|0\rangle+\beta|1\rangle$, where $|\alpha|^{2}+|\beta|^{2}=1$. Initially, Alice the sender, combines the unknown qubit state, with the EPR pair and performs a Bell measurement, and conveys the outcome of her measurement to Bob the receiver, via two classical bits of information. Bob, then performs appropriate unitary transformations on his particles and obtains the unknown qubit information. Hence, the resources required to achieve this are one ebit of entanglement and two classical bits of information. Recently, attention has turned towards the teleportation of an arbitrary two qubit state [3, 4, [5, [6] given by :

$$
|\psi\rangle=\alpha|00\rangle+\gamma|10\rangle+\beta|01\rangle+\delta|11\rangle,
$$

where, $|\alpha|^{2}+|\beta|^{2}+|\gamma|^{2}+|\mu|^{2}=1$. The resource used for teleporting this are two ebits and four classical bits.

Quantum information splitting or secret sharing is the splitting up of quantum information between various parties, such that if only each of them cooperate, one of them can obtain the desired information. Hillery et al. 7], have demonstrated the sharing of an unknown single qubit of information among three parties using the three and the four particle GHZ states. The scheme uses, two cbits of information resources. Recently, QIS of an arbitrary two qubit state was proposed by two of the present authors, utilizing the five particle Brown and cluster states [5, 8]. The procedure utilizes four cbits as information resource. In all these schemes, the state that is to be shared is unknown to the sender. However, in many practical scenarios, the information that is to be shared may be known to the sender. In such cases, the classical information resource can be greatly reduced and need not be wasted. Recently, it was shown that if the sender knows the single qubit information that she is sending, it could be split among two parties by utilizing just one classical bit [9]. In this paper, we extend these results to an arbitrary two qubit state by investigating

\footnotetext{
*Electronic address: sreraman@loyolacollege.edu

†Electronic address: prasanta@prl.res.in
} 
the five particle cluster and the Brown state as entangled resources. Let Alice, possess the two qubit information, in Eq.(1) which she wants Bob and Charlie to share and be aware of the information. Here, $\alpha$ is real, while the other coefficients are complex. If we choose, $\alpha=\gamma=\frac{1}{2}, \beta=\delta=\frac{1}{2} e^{i \phi}$, then $|\psi\rangle$ is analogous to the equatorial state on the Bloch sphere. It is to be noted that, if $\phi=0$ or $\pi$, then all the coefficients are real, in which case $|\psi\rangle$ is called a real state.

\section{CLUSTER STATE FOR QIS OF A TWO QUBIT STATE}

The five particle cluster state is given by,

$$
\left|C_{5}\right\rangle=\frac{1}{2}(|00000\rangle+|00111\rangle+|11101\rangle+|11010\rangle)
$$

There are two ebits of entanglement between pairs $15 \mid 234$. Hence, we let Alice posses qubits, 1 and 5 and Bob possess the remaining qubits. Since, Alice is aware of the information that is to be sent, she can perform a two particle measurement in the basis involving the coefficients of the input state. The outcome of the measurement performed by Alice and the state obtained by Bob and Charlie are shown in the table below

TABLE I: The outcome of the measurement performed by Alice and the state obtained by Bob and Charlie

\begin{tabular}{|c|c|}
\hline Outcome of the measurement & State obtained \\
$\frac{1}{2}(\alpha|00\rangle+\beta|01\rangle+\gamma|10\rangle+\delta|11\rangle)$ & $\alpha|000\rangle+\beta^{*}|011\rangle+\gamma|101\rangle+\delta^{*}|110\rangle$ \\
$\frac{1}{2}\left(\beta^{*}|00\rangle-\alpha|01\rangle+\delta^{*}|10\rangle-\gamma|11\rangle\right)$ & $\beta|000\rangle-\alpha|011\rangle+\delta|101\rangle-\gamma|110\rangle$ \\
$\frac{1}{2}\left(\gamma|00\rangle-\delta^{*}|01\rangle-\alpha|10\rangle+\beta^{*}|11\rangle\right)$ & $\gamma|000\rangle-\delta|011\rangle-\alpha|101\rangle+\beta|110\rangle$ \\
$\frac{1}{2}\left(\delta^{*}|00\rangle+\gamma|01\rangle-\beta^{*}|10\rangle-\alpha|11\rangle\right)$ & $\delta|000\rangle+\gamma|011\rangle-\beta|101\rangle-\alpha|110\rangle$ \\
\hline
\end{tabular}

Neither Bob nor Charlie can obtain the information through local operations on their own qubits. Alice encodes the outcome of her measurement in classical bits and sends it to Charlie. Bob can now perform a single particle measurement and send the outcome of her measurement to Charlie. Each of the measurement can be performed with equal probability $\left(=\frac{1}{4}\right)$. If Alice performs a measurement in the first basis, it is not possible for Charlie to obtain the desired state, unless the information to be shared is equatorial or real qubit states. If Alice chooses to perform a measurement in remaining members of the basis, then Charlie can obtain the desired state after receiving Bob's measurement output as in the standard QIS scheme. In this sense, the present algorithm is neither fully deterministic nor probabilistic. Now, Bob can perform a measurement in the basis $\frac{1}{\sqrt{2}}(|0\rangle+|1\rangle$, then Charlie's system collapses to $\left.\frac{1}{2}(00\rangle+e^{-i \phi}|11\rangle \pm|01\rangle \pm e^{-i \phi}|10\rangle\right)$, To correct the phase factor, Charlie has to use the gate controlled phase of $e^{(2 i \phi)}$ i.e., when the first qubit is $|1\rangle$ then a phase of $e^{2 i \phi}$ is introduced.

In the original scheme, using cluster state for QIS of an arbitrary two qubit state, Alice performs a four particle measurement and conveys the outcome of her measurement to Charlie via four cbits of information. In comparison, the present scheme uses only two cbits of information, if the two qubit state is chosen from partially known equatorial or real states. Hence for these special states this procedure reduces measurement complexity and the consumption of classical information by two cbits.

\section{BROWN STATE FOR QIS OF A TWO QUBIT STATE}

The Brown state is given by

$$
\left|\psi_{5}\right\rangle=\frac{1}{2}\left(|001\rangle\left|\phi_{-}\right\rangle+|010\rangle\left|\psi_{-}\right\rangle+|100\rangle\left|\phi_{+}\right\rangle+|111\rangle\left|\psi_{+}\right\rangle\right),
$$

where, $\left|\psi_{ \pm}\right\rangle=\frac{1}{\sqrt{2}}(|00\rangle \pm|11\rangle)$ and $\left|\phi_{ \pm}\right\rangle=\frac{1}{\sqrt{2}}(|01\rangle \pm|10\rangle)$ are Bell states. In this scheme, we let Alice have first two particles, Bob have the third one and Charlie the last two particles. As in the previous case, in this scheme also Alice can perform a measurement in appropriate basis. The outcome of the measurement performed by Alice and the Bob-Charlie system is shown below. 
TABLE II: The outcome of the measurement performed by Alice and the state obtained by Bob and Charlie

\begin{tabular}{|c|c|}
\hline Outcome of the measurement & State obtained \\
$\frac{1}{2}(\alpha|00\rangle+\beta|01\rangle+\gamma|10\rangle+\delta|11\rangle)$ & $\alpha\left|\eta_{1}\right\rangle+\beta^{*}\left|\eta_{2}\right\rangle+\gamma\left|\eta_{3}\right\rangle+\delta^{*}\left|\eta_{4}\right\rangle$ \\
$\frac{1}{2}\left(\beta^{*}|00\rangle-\alpha|01\rangle+\delta^{*}|10\rangle-\gamma|11\rangle\right)$ & $\beta\left|\eta_{1}\right\rangle+\alpha\left|\eta_{2}\right\rangle+\delta\left|\eta_{3}\right\rangle-\gamma\left|\eta_{4}\right\rangle$ \\
$\frac{1}{2}\left(\gamma|00\rangle-\delta^{*}|01\rangle-\alpha|10\rangle+\beta^{*}|11\rangle\right)$ & $\gamma\left|\eta_{1}\right\rangle-\delta\left|\eta_{2}\right\rangle-\alpha\left|\eta_{3}\right\rangle+\beta\left|\eta_{4}\right\rangle$ \\
$\frac{1}{2}\left(\delta^{*}|00\rangle+\gamma|01\rangle-\beta^{*}|10\rangle-\alpha|11\rangle\right)$ & $\delta\left|\eta_{1}\right\rangle+\gamma\left|\eta_{2}\right\rangle-\beta\left|\eta_{3}\right\rangle-\alpha\left|\eta_{4}\right\rangle$ \\
\hline
\end{tabular}

Here,

$$
\begin{aligned}
\left|\eta_{1}\right\rangle & =\frac{1}{2}(|101\rangle-|110\rangle), \\
\left|\eta_{2}\right\rangle & =\frac{1}{2}(|000\rangle-|011\rangle), \\
\left|\eta_{3}\right\rangle & =\frac{1}{2}(|001\rangle+|010\rangle), \\
\left|\eta_{4}\right\rangle & =\frac{1}{2}(|100\rangle+|111\rangle) .
\end{aligned}
$$

Alice can encode the outcome of her measurement in two cbits and send it to Charlie. As in the previous case, even here, if the first member of the set is chosen as the measurement basis, then one can obtain the desired information, only if the initial state is either equatorial or real. Bob then performs a measurement in the basis $\frac{1}{2}(|0\rangle \pm|1\rangle)$. The corresponding states obtained by Charlie are :

$$
\left.\pm(01\rangle-|10\rangle)+e^{-i \phi}(|00\rangle-|11\rangle)+(|01\rangle+|10\rangle) \pm e^{-i \phi}(|00\rangle+|11\rangle)\right]
$$

on which Charlie applies a suitable unitary operator to obtain the state. Hence, the protocol suceeds.

\section{CONCLUSION}

In conclusion, we have shown that, for the QIS of a two qubit state, the classical information resource is reduced by half, if the two qubit state is equatorial or real. Hence, one need not waste classical information resource, when some information is known about the initial state. Interestingly, the scheme is neither fully probabilistic nor deterministic. It is worth checking, if classical or quantum information resources in other protocols can be reduced when some information about the initial state is known to the sender. Generalization to the QIS of real or equatorial $N$ qubit states using $2 N$ and a $(2 N+1)$ qubit states as an entangled resource needs to be explored.

[1] M. A. Nielsen and I. L. Chuang, Quantum Computation and Quantum Information, (Cambridge Univ. Press, 2002).

[2] C. H. Bennett, G. Brassard, C. Crepeau, R. Jozsa, A. Peres, and W. K. Wootters, Phys. Rev. Lett. 70, 1895 (1993).

[3] G. Rigolin, Phys. Rev. A 71, 032303 (2005).

[4] Y. Yeo and W. K. Chua, Phys. Rev. Lett. 96, 060502 (2006).

[5] S. Muralidharan and P. K. Panigrahi, Phys. Rev. A 77, 032321 (2008).

[6] S. Muralidharan and P. K. Panigrahi, eprint quant-ph/0802.3484.

[7] M. Hillery, V. Buzek, and A. Berthiaume, Phys. Rev. A 59, 1829 (1999).

[8] S. Muralidharan and P. K. Panigrahi, eprint quant-ph/0802.0781.

[9] Z. J. Zhang and C. Y. Cheung, J. Phys. B: At. Mol. Opt. Phys. 41, 015503 (2008). 